\title{
ANISOTROPIC EFFECTS IN THOMSON CASCADE MODELS OF ACTIVE GALACTIC NUCLEI
}

\author{
MATTHEW G. BARING* \\ NASA Goddard Space Flight Center, \\ Lab. for High Energy Astrophysics, Code 665, \\ Greenbelt, MD 20771, U.S.A.
}

\begin{abstract}
CGRO observations of high energy $\gamma$-rays from active galactic nuclei (AGN) suggest that beaming may be prevalent in these sources. Anisotropic effects in AGN continuum spectra produced by the inverse Compton scattering mechanism are outlined, in particular the resulting spectral breaks and modification of spectral indices that depend strongly on the electron anisotropy and the observational viewing perspective.
\end{abstract}

\section{Thomson Cascade Spectra}

The recent EGRET observations of energetic $\gamma$-rays coming from blazars, together with the Whipple detection of TeV $\gamma$-rays from Mkn421 has bolstered support for the hypothesis that many active galactic nuclei are strongly beamed sources. These high energy data contrast the turnovers seen (or inferred) by other instruments aboard the Compton Gamma-Ray Observatory (CGRO) in Seyferts, indicating either that they are possibly a distinct class of AGNs, or perhaps that observational orientation may provide the difference between hard and softer emitters. Prior to the launch of CGRO, pair cascade models of AGNs largely focussed in Seyferttype spectra, generating the continuum through inverse Compton scattering of UV photons by isotropic relativistic electrons. The new observations indicate that this electron injection is probably beamed in many sources; some of the consequences of such anisotropic injections are outlined here.

Moderate degrees of beaming suppress two-photon pair production to the point that the cascades can be mediated purely by Compton scattering. In such situations, collisions between relativistic electron beams and soft photons can generate flatter spectra at X-ray energies near the direction of beaming but with much lower intensity than for isotropic electrons. Spectral steepening by an index of order unity occurs at soft gamma-ray energies, for beaming angles of a few degrees, above which the intensity approximates the isotropic case. Steeper, softer spectra are seen at large viewing angles relative to the beam axis. Solutions obtained to the photon kinetic equation, in cases where multiple scatterings of the photons occur, reveal that electron beaming can generate spectra steeper than the familiar isotropic case, since energy gains in successive upscatterings are reduced once the photons become beamed quasi-parallel to the electrons. These electron anisotropy effects may well help to determine AGN beaming and viewing angles from refined spectral models of hard X-ray and gamma-ray emission.

* NAS-NRC Research Associate.

T. J.-L. Courvoisier and A. Blecha: Multi-Wavelength Continuum Emission of AGN, 343.

(C) 1994 IAU. Printed in the Netherlands. 